Itinéraires Itinéraires

Littérature, textes, cultures

2009-2 | 2009

Caraïbe et océan Indien

\title{
Édouard Glissant et Derek Walcott : une vision fragmentée de l'histoire
}

Aminata Keita

\section{(2) OpenEdition}

\section{Journals}

Édition électronique

URL : http://journals.openedition.org/itineraires/246

DOI : 10.4000/itineraires.246

ISSN : 2427-920X

Éditeur

Pléiade

\section{Édition imprimée}

Date de publication : 1 juillet 2009

Pagination : 33-46

ISBN : 978-2-296-09639-4

ISSN : 2100-1340

\section{Référence électronique}

Aminata Keita, «Édouard Glissant et Derek Walcott : une vision fragmentée de l'histoire ", Itinéraires [En ligne], 2009-2 | 2009, mis en ligne le 02 juin 2014, consulté le 21 décembre 2020. URL : http:// journals.openedition.org/itineraires/246 ; DOI : https://doi.org/10.4000/itineraires.246

Ce document a été généré automatiquement le 21 décembre 2020.

\section{cc)}

Itinéraires est mis à disposition selon les termes de la licence Creative Commons Attribution - Pas d'Utilisation Commerciale - Pas de Modification 4.0 International. 


\title{
Édouard Glissant et Derek Walcott : une vision fragmentée de l'histoire
}

\author{
Aminata Keita
}

Un homme vit la moitié de sa vie

La seconde moitié est mémoire.

Derek Walcott

1 L'interprétation de la mémoire historique peut prêter à diverses ambiguïtés référentielles, si bien qu'elle n'a de cesse d'alimenter un grand nombre de controverses. En effet, le travail de mémoire suppose une multitude d'images et d'éléments : souvenirs, oubli, reconnaissance morale... C'est aussi le regard des témoins, des survivants et des descendants qui se heurte en permanence aux horreurs de l'humanité, aux événements menacés de disparition ou totalement absents de la mémoire collective. Dès lors, certains écrivains ont cherché à mettre au jour ce passé disparu, à rendre inoubliables ces blessures afin de reconquérir une nouvelle dignité humaine. Édouard Glissant et Derek Walcott ont fait de la question historique une thématique privilégiée de leurs œuvres. L'histoire de leur peuple s'inscrit dans de nombreux textes de leur production littéraire. Pour ces auteurs, la question de la mémoire est d'autant plus importante que l'histoire des Antilles est souvent qualifiée d'histoire d'emprunt, puisqu'elle est calquée sur une histoire préalablement élaborée par les colons anglais ou français. Or, le discours colonial n'interroge nullement la parole des esclaves noirs. Grâce à quelques extraits d'œuvres poétiques, il s'agira de dégager les différentes articulations d'une poétique de l'histoire et les conditions d'une possible lecture de la mémoire. Il s'agira de montrer entre autres comment transparaissent la dimension d'une autobiographie poétique et les apories d'une écriture mémorielle soucieuse de déjouer les manipulations historiques.

\section{Création poétique et réalité historique}

2 La mémoire et la transmission des faits historiques constituent les problèmes majeurs auxquels tout Antillais est confronté, dans la mesure où l'oubli du passé ancestral 
pourrait être à ce jour l'une des premières causes d'un malaise identitaire. Édouard Glissant parle "d'absence noire ", voire de "non-histoire », pour désigner la mémoire antillaise et négro-africaine atrophiée. Cette mémoire s'est manifestement altérée après trois siècles d'esclavage et de colonisation. C'est donc par le moyen de l'écriture et plus particulièrement celui de la poésie qu'il va tenter de fonder son entreprise mémorielle comme il l'énonce en ces termes :

Au nom ici du démarquage poétique, pour témoigner d'une splendeur morale. Je m'oblige à saluer : l'homme ce lumineux désir de chant. Vocatif, trop.

(Glissant, 1994 : 28)

3 Partant du projet de réinvestir le passé et de reconstruire une autre mémoire, Glissant formule quelques principes qu'il serait intéressant de préciser. Il utilise d'abord un grand nombre d'éléments qui suggèrent une écriture autobiographique, comme le discours à la première personne, pour évoquer son vécu ainsi que celui de ses proches. Puis, comme si le poète se trouvait en quête d'un confident, la représentation de l'altérité commence à prendre forme dans la deuxième personne du singulier «tu» et du pluriel «vous", comme dans les premiers vers du recueil Le sang rivé:

Or je suis dans l'histoire jusqu'à la moindre moelle

Séculairement installé : en ce midi que je disais

fort comme l'ignorance : elle roule en ses graviers...

Homme coolie-marin mer-mousse

Ah la rosée de mes cheveux que tu as cru une poussée de coraux morves...

(Ibid. : 27)

4 L'emploi de la gradation dans le discours montre que le poète expose à ses compatriotes le projet de récupérer leur mémoire. En effet, étant donné que chaque Antillais semble atteint par les effets de l'histoire, qu'il en soit conscient ou non, l'expérience mémorielle du poète devient collective, comme si le peuple s'associait à Glissant pour sauver sa culture de l'amnésie, d'où l'affirmation:

C'est un pays qui bat des hanches contre l'aveuglement

Races races cannes sagaies...

(Ibid. : 26)

5 Ainsi, la mémoire se présente comme étant de l'ordre de l'intime puis elle s'ouvre vers le collectif. C'est ce dernier aspect qui semble assimiler le rôle du poète à celui de l'historien. On assiste alors à une sorte de plurivocité dans les différents poèmes: l'écriture ne se recentre plus sur le moi du poète mais bien sur une communauté donnée et sur toutes les catégories d'énonciation. Quant à l'objet de la pratique mémorielle, il est tourné pour une large part vers l'esclavage, et cela parait d'autant plus légitime qu'il représente l'événement fondateur de la société antillaise. L'idée de se projeter dans une telle remémoration relève donc d'une entreprise de réappropriation identitaire. Il s'agit de reconstruire de nouvelles données susceptibles de faire sens pour tout le monde. Pour ce faire le poète va faire appel à des modalités particulières que Philippe Lejeune nomme des « unités de départ » (Lejeune, $200^{1}: 249$ ).

\section{L'aveu à partir de l'imagination}

6 Les poèmes d'Édouard Glissant et ceux de Derek Walcott fonctionnent comme des « témoignages » élaborés à partir des capacités créatrices de la poésie. L'imagination en est l'une des principales ressources et elle constitue la première " unité de départ » du travail de remémoration. Cela peut paraître contradictoire dans la mesure où le travail 
autobiographique suppose une sincérité absolue mais le rêve et l'imagination des auteurs ont la même valeur que les autres modèles de descriptions utilisés dans le récit autobiographique classique. En réalité, les Antilles n'ont pas de véritables livres d'histoire, de grandes fresques historiques qui prennent en compte la souffrance endurée par les esclaves noirs venus d'Afrique dès le $\mathrm{XVI}^{\mathrm{e}}$ siècle. C'est pourquoi les poètes contemporains se donnent pour mission la remémoration de l'histoire de leurs origines, notamment à travers le songe, d'où le titre d'un recueil de Glissant Pays rêvé, pays réel: le " pays rêvé » représente l'Afrique et, loin d'être un fantasme personnel, cette évocation permet d'avoir une vision rétrospective des ancêtres dont il est séparé depuis plusieurs générations. D'ailleurs, lorsqu'il évoque la rupture opérée par le voyage de ses ancêtres de l'Afrique aux îles Caraïbes, c'est en recourant à l'image du rapt. Il reconstitue toutes les images du "pays d'avant» que lui ont proposées ses lectures ou les récits oraux. On retrouve alors des éléments de la réalité africaine dans l'ensemble de l'œuvre, comme « le tambourin des baobabs", "les sables de déserts de brousse, les barrissements", "les cannes sagaies ». De même, lorsque Glissant décide de rendre compte de l'expérience de la traite négrière, c'est à travers l'image de l'île de Gorée, ancien comptoir négrier en Afrique. Gorée suppose ici l'imprévisible déchirure avec le pays d'origine, le pays des ancêtres qu'il traduit en ces termes :

Il n'eut l'espace de héler dépassement, ayant drivé entre rive et haut bord, dans l'île

d'amarrage où les rêves d'hier tuent au garrot les rêves de demain. (Glissant, 1994 :

245)

7 Au fil des textes, la poésie de Glissant ne cherche plus à décrire simplement les événements passés, elle devient elle-même représentation de la réalité, et comme s'il nouait un pacte avec son lecteur, Glissant tente de mimer l'entassement et la douleur des esclaves à bord des bateaux négriers, par des procédés spécifiques ou des combinaisons particulières, comme l'écriture verticale du poème «Brûlis » :

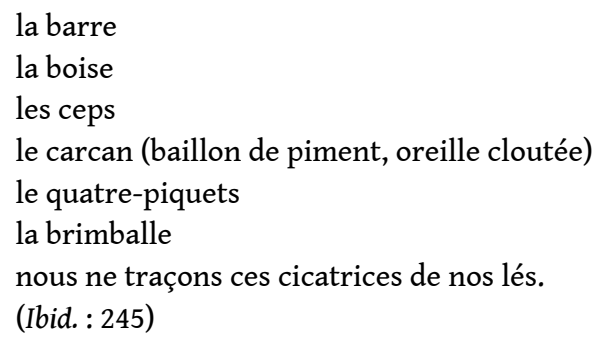

8 L'emploi de termes évocateurs, comme la «brimbale » ou le «quatre-piquets » qui sont autant de mots vieillis, tente de réactualiser la souffrance endurée pendant la traversée. Par ailleurs, le texte ne se limite pas seulement à cette sorte de fantasme personnel, il prend peu à peu la forme d'une "terre intérieure", puisqu'on retrouve un certain nombre de vers qui crée un rapport privilégié entre la mémoire et les paysages.

De son côté, Derek Walcott appelle à une relecture de l'histoire mais le poète saint-lucien refuse quant à lui de sombrer dans la nostalgie et de revendiquer la découverte du passé précolonial. Bien au contraire, il montre à plusieurs occasions un certain dégoût pour l'histoire. Il préconise de tourner le dos au passé et de s'orienter plutôt vers le futur. À l'inverse d'Édouard Glissant, il n'évoque que très rarement le continent africain ; la voie du retour à la Mère Afrique longtemps prôné par les chantres de la négritude et ceux du mouvement afro-américain Harlem Renaissance n'a guère de sens pour lui. Certes, ses poèmes sont imprégnés de références culturelles historiques mais ces dernières revêtent davantage une fonction illustrative. Ses poèmes évoquent une sorte de spectre qui vient 
hanter les générations présentes. Dans le célèbre poème "The star apple-kingdom ", Derek Walcott introduit une allégorie du rapport à l'histoire à travers la figure d'une femme, une "beauté noire ", conquérante, "chasseresse » venue d'un passé lointain et qui se veut représentative de la "Revolution »: "I am the darker, the older America ». Cette femme est d'abord présentée comme un être attirant, plein de mystère voire enchanteur mais dont la tentative de séduction se transforme très vite en échec :

Until a black woman, shawled like a blizzard,

Climbed up the stairs and knocked at the door

of this dream, whispering in the ear of the keyhole:

"Let me in, I'm finished with praying, I'm the Revolution.

I am the darker, the older America."

She was as beautiful as a stone in the sunrise,

Her voice had the gutturals of machine guns

(Walcott, 1992a : 94)

La résistance du poète aux avances de cette muse de l'histoire est aussi le rejet d'une forme d'inspiration littéraire qui voudrait donner à la littérature la responsabilité totale d'une idéologie purement fondée sur le passé historique :

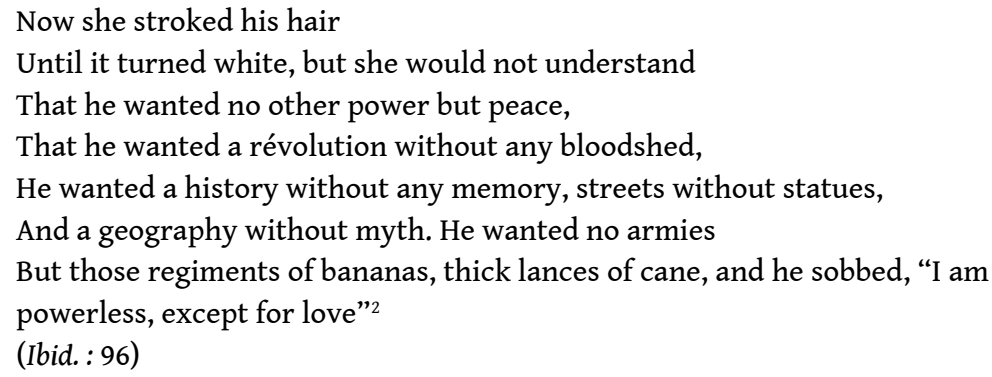

Si Derek Walcott se refuse à situer son discours à l'intérieur de l'histoire, il ne peut toutefois pas faire fi de son incessant appel. La seule alternative pour lui est de réagir avec vigilance: il adopte des dispositions particulières afin de pouvoir émerger des vagues de l'histoire dès que le besoin se fait sentir. Ainsi lorsqu'il écrit «The sea is history " (Walcott, 1992b: 48), il rappelle à l'instar d'autres écrivains antillais que la mémoire de tout Antillais est contenue dans la mer qui borde l'île et il prend acte des épisodes douloureux de la traversée des esclaves dans les cales des bateaux négriers. Mais il s'inspire aussi d'autres modèles littéraires comme Ulysse ou Robinson Crusoé.

\section{L'aveu à partir de l'espace}

Loin de n'être qu'un phénomène chronologique, la mémoire est également question de spatialité. Tous les procédés mnémotechniques font appel à l'espace, de sorte qu'on peut qualifier la reconstitution de la mémoire antillaise d'écriture "archipélique » (Glissant). Les espaces maritimes et terrestres structurent les poèmes. Les uns et les autres sont assimilés, d'autant que la mer est à l'histoire caribéenne ce que la terre est à l'histoire des origines si l'on se réfère à la Genèse.

Maintenant les sables sont d'autre clarté. Il faut choisir, Il faut venir! Soit par la mer, connue des marins-pêcheurs aux songes funèbres, soit dans la terre, tronc noir et nu... Et puis, la saviez-vous, cette entreprise de bâtir le paysage ? - Parfois le cœur est écrasé, l'air est hostile ; parfois la main s'apaise - et la lumière monte des choses comme une parole d'architecte. (Glissant, 1994 : 71) 
13 Tout en se rattachant à la vision d'images locales et à la géographie notamment, Édouard Glissant et Derek Walcott nourrissent leur poétique de toutes les références topographiques qui les entourent. À partir du paysage, ils créent « une problématique du lieu et du lien » (Jacquart, 1992 : 229), ce qui leur permet d'exprimer leur histoire, leurs origines, leur identité. Cette géographie offre à son tour toutes les réponses possibles et relatives à l'histoire. D'ailleurs, de nombreux titres de recueils ou même de sujets traités dans la production littéraire d'Édouard Glissant accordent une large place à la notion d'espace: Terre inquiète, Les Indes, Le sel noir, Pays rêvé, pays réel... Dans le poème «The schooner flight » de Derek Walcott, la première lecture dévoile la présence des images de la mer et des îles des Caraibes. Le bruit lent et lancinant de la mer domine à la fois dans un état brut et légèrement surréel. Édouard Glissant, lui, profite de cette opportunité pour s'abandonner au lyrisme grâce auquel il fait parler tous les éléments de la nature, en particulier la végétation tropicale à l'exemple des forêts montagneuses du Nord de la Martinique mentionnées dans cet extrait :

La forêt subitement hurle à la vie. Les étoiles, rôdeuses, envahissent les écluses. Vivante ô vivante, reine. Tes pieds vont le chemin, manguiers abandonnés. Ta peau retournée est un labour rouge. Vivante. (Glissant, $1994: 28$ )

14 Même si la nature insulaire est perçue dans toute sa beauté, celle que lui reconnaît généralement le touriste, il n'empêche qu'Édouard Glissant s'efforce d'y retrouver les vestiges, les ruines de l'histoire. Le poète réhabilite des figures mal dessinées par l'historiographie officielle, en l'occurrence celle du nègre-marron, cet esclave fugitif et réfractaire au système plantationnaire. Le poète accorde ici une grande importance au marronnage, dans la mesure où cette expérience fut pendant longtemps réfutée par les historiographes comme le mode d'opposition le plus radical du point de vue social, culturel et politique adopté par les esclaves. L'exil des marrons a permis la formation de sociétés parallèles, d'où les nombreuses personnifications accordées aux végétaux dans le vers « la forêt subitement hurle à la vie ». D’ailleurs, ces sociétés ont engendré à certaines périodes de l'histoire des Antilles, et en particulier à Cuba et à la Jamaïque, des révoltes au nom de leur liberté, comme l'a illustré Alejo Carpentier dans Le Royaume de ce monde.

Loin d'être un simple décor, la vision du paysage archipélique permet d'activer les traces premières, le massacre des autochtones, les Amérindiens, la traite des esclaves dès le début $\mathrm{du} \mathrm{XVI}{ }^{\mathrm{e}}$ siècle et l'arrivée des Indiens au XIX ${ }^{\mathrm{e}}$ siècle dans le sud de l'archipel. Pour les auteurs, le paysage représente une sorte de monument historique muet, au point qu'ils n'hésitent pas à inscrire leurs poèmes dans une sorte de géographie intime. La tâche revient alors au lecteur de distinguer les souvenirs douloureux que peuvent inspirer ces paysages de l'image idyllique véhiculée par certaines affiches publicitaires. On peut alors penser que la profonde tristesse qui vient se fondre dans le discours poétique des auteurs n'est autre qu'une réaction contre ce constat débilitant. Derek Walcott propose aux artistes de « remonter à travers les ténèbres vers l'amnésie finale qui les attend. Ce gouffre obscur qui s'ouvre sous leur pas est terrifiant. C'est la remontée de l'homme vers le singe. Il faudrait que chaque acteur accomplisse ce voyage pour rejoindre ces origines" (Walcott, 2004a: 10). La poésie de Derek Walcott tente inlassablement de rompre avec les images ou la gamme de couleurs stéréotypées des îles antillaises au profit de représentations beaucoup plus contrastées, à la fois lumineuses et sombres ou noires qui sont celles de l'histoire. Le poète crée ainsi un pont entre ses deux passions, l'écriture et la peinture aquarelle. 

Camille Pissarro, a permis de réaffirmer, auprès des lecteurs francophones, l'intérêt que le poète saint-lucien porte à la création plastique. Dès les premières pages du Chien de Tiepolo, on note une formulation évocatrice :

Ce qui doit être vrai de la vie remémorée

C'est la fraîcheur du détail : voilà comment c'était

l'odeur d'amande qu'exhale la feuille arrachée à l'amandier, ton visage vernissé par les embruns quand explosent les vagues.

(Walcott, 2004b : 19)

C'est donc cette «fraîcheur du détail » qu'on retrouve tout au long des poèmes walcottiens, présentant tour à tour un développement explicite et une longue énumération qui permettent à chaque moment de relancer l'interprétation de telle ou telle séquence de l'histoire. Walcott s'applique à façonner chaque mot, chaque vers, chaque strophe, afin de lui donner toute la capacité de figuration qui lui permet de dépasser son aspect littéraire et de rejoindre le monde réel.

Édouard Glissant, pour sa part, met également l'accent sur la représentation du paysage apte à dévoiler l'histoire en plongeant au cœur des vérités ancestrales. S'il ne manie pas le pinceau comme Walcott, il réactualise la mémoire collective en donnant un sens particulièrement fort à la métaphore. C'est ainsi qu'on peut voir l'adjectif «noir » associé à plusieurs éléments de la nature: "sable noir ", "plage noire ", "sel noir », "fumée noire ", «tibias noirs ", etc. Dans l'évocation de la couleur sombre « noir », le lecteur est invité à percevoir les traces laissées par la traversée des esclaves, lesquelles hantent le poète comme un lancinant cauchemar.

\section{Les apories de l'écriture mémorielle}

Ainsi je fus colonie d'enfants martyrs de chiens trouvés de squales non convertis... La pauvreté est ignorance de la terre, l'imaginé est passion... Racines, racines, je n'en finirai pas de tirer sur vos mamelles fécondes... (Glissant, $1994: 28$ )

Édouard Glissant et Derek Walcott recomposent, certes, la mémoire du peuple antillais mais ils ne reconstituent pas les faits de manière strictement rétrospective ou linéaire. $\mathrm{Au}$ lieu de cela, c'est un discours historique sous une forme éclatée et parfois cyclique qu'on retrouve à la fois dans le corpus poétique et romanesque chez Édouard Glissant. Il est à noter que ses romans sont parfois à la limite du roman à énigme ou du roman poétique comme si le discours romanesque prenait le risque de reproduire la problématique que pose le passage du temps face à l'élaboration d'une mémoire collective. Les mêmes thèmes, les mêmes scènes, les mêmes images, et parfois les mêmes noms reviennent sans cesse afin d'être réajustés dans la production littéraire d'Édouard Glissant. Les souvenirs sont très souvent présentés de façon autonome et juxtaposés les uns aux autres. Ainsi, les faits ne sont pas livrés ou racontés dans un ordre strict mais par fragments ou par àcoups, même si dans l'ensemble on retrouve des faits de même nature ou de même type. On note plusieurs événements précis, datés et référencés dans les archives : le massacre des Amérindiens - évoqué par "Chabin", le double de Derek Walcott dans "The schooner flight " lorsqu'il accède aux portes de la Dominique -, ou encore la bataille navale des Saintes - cette longue lutte qui opposa la flotte anglaise de l'amiral Rodney à la flotte française de l'amiral de Grasse pour la domination de certaines îles dont celle de Sainte-Lucie en 1782 :

Itinéraires, 2009-2 | 2013 


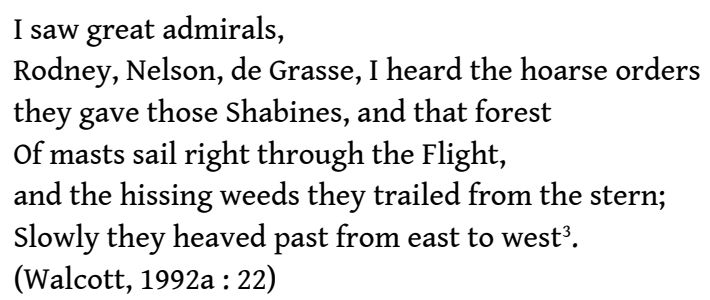

Ce temps chronologique sert de toile de fond à une temporalité plus fragmentée et littéraire. Derek Walcott superpose le passé au présent en racontant des faits historiques comme s'ils se situaient dans un temps encore présent ou dans la réalité insulaire. Édouard Glissant résume cette subjectivité temporelle par l'appellation: "vision prophétique du passé » (Glissant, 1981: 132), ce qui lui permet d'actualiser sa mémoire ainsi que celle de ses aïeux.

En effet, le temps historique ou calendaire, propre à l'ancienne puissance colonisatrice, a mis en exergue des blancs et des silences ; l'idée des auteurs n'est autre que de réinventer une nouvelle périodisation. Édouard Glissant se plaît à évoquer «l'absence noire » en guise de réponse à l'histoire officielle qui a volontairement laissé des trous dans son tissu narratif.

La démarche des auteurs s'apparente donc à celle du philosophe allemand Walter Benjamin qui invite à " prendre l'histoire à rebrousse poil » (Benjamin, $2000: 433)$ au lieu de se laisser porter par elle. Aussi les auteurs cherchent-ils à rompre avec l'historiographie officielle ou encore avec l'idée d'un continuum dans la mesure où la reconstitution historique établie par la métropole n'a pas permis à l'Antillais de véritablement appréhender sa propre histoire et de pouvoir l'intérioriser. On assiste donc à un mouvement d'anamnèse, à une recherche du passé et à une mise à jour de ce qui a été nivelé par le processus de colonisation. Dans son roman La Case du commandeur où il est également question du rapport à la mémoire, Édouard Glissant illustre la contrelinéarité du temps historique en proposant de «sauter de roche en roche dans ce temps incertain!» (Glissant, 1997 : 118). C'est d'ailleurs «ce temps incertain» qui justifie le caractère épars et fragmentaire de son écriture.

\section{La fragmentation}

À l'idée de la fragmentation, Daniel-Henri Pageaux associe « le souci de diviser, de réduire pour mieux faire consommer et transformer une partie en un fragment tout à la fois positif, beau, assimilable» (Pageaux, 2003: 211). Dans le but de reproduire toute la dimension profonde de la mémoire, Édouard Glissant accorde une importance particulière à la forme de son art. Dans Poèmes complets, le lecteur prend contact avec l'œuvre à partir de ce qu'il y a de plus mouvant et de plus dynamique comme les jeux structuraux sur la fragmentation. Étant donné que le temps historique offre une histoire éclatée, faite de nombreux silences et de trous, l'histoire du poète devient un montage et chaque poème se présente comme un fragment de réalité historique, où le passage d'un épisode à un autre se fait sans lien de cause à effet. On assiste alors à une répartition des différents poèmes qui s'effectue à partir de blancs typographiques, de numéros ou de titres. Tous ces éléments révèlent un savoir dans un ordre brisé et permettent de suivre les jeux complexes de la mémoire. Pour Édouard Glissant, la poésie devient alors une matérialisation, une cristallisation de l'histoire où chaque strophe en résonance avec le passé est livrée de manière éparse, sous forme d'éclats, comme une terre qu'on ravage et 
qu'il s'agit par la suite d'assembler pour en conserver le souvenir et lui donner une identité.

\section{Quelle réécriture pour une histoire inarchivée?}

Pour nombre d'écrivains postcoloniaux, la mise à jour de la mémoire collective de leur peuple constitue une urgence. Derek Walcott et Édouard Glissant, pour leur part, vont tenter de mettre leur plume au service d'une relecture, d'une réinterprétation et d'une réécriture de l'histoire. Les auteurs reconstruisent des événements passés, et cette reconstitution s'effectue à partir d'un matériau textuel déjà existant. On peut parler de «transdiégétisation» (Genette, 1992: 420). L'objectif d'une telle méthode consiste à traiter et à transformer le matériau textuel afin de le faire ressembler à un matériau déjà existant. Ce travail de réécriture est un des objectifs visés par Édouard Glissant. Aussi, en recourant à des jeux sur le signifiant et en usant de métaphores, le poète procède à une sorte d'alchimie sur le langage, afin d'extraire d'autres secrets du passé. En reprenant le principe de la transdiégétisation, il s'approprie la destruction de Carthage dans Le Sel noir, ce qui lui permet de pallier certains oublis et de susciter un dynamisme dans sa représentation historique en recourant à des images symboliques fortes de sens. Pour lui, l'épisode historique de Carthage permet de remonter au premier massacre d'une ville africaine en plein essor :

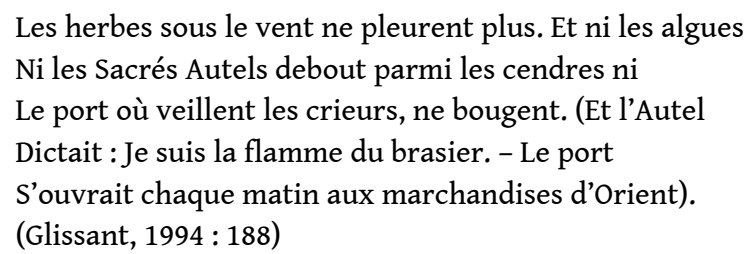

Ce processus de transdiégétisation permet à Édouard Glissant d'opérer un glissement de sens, puisqu'on peut voir en cet épisode historique un événement prémonitoire. Le pillage et la ruine de Carthage en Afrique sous le pouvoir de «Scipion ", personnification de l'Europe, annoncent en quelque sorte les tragédies à venir de l'Afrique, comme l'esclavage et la déportation :

Comme à Précieuse évanouie on crie du Sel, Scipion Blafard crie vers ton âme qu'il bafoue. Laisseras-tu Ce triste méditer les déserts nés de toi, disant: Comme est paisible mon repos sur ce lit de terreurs Puis il goûte le sel, pensant aux Jeux qu'il donnera.

(Ibid. : 189)

En s'appuyant sur cette référence historique, Glissant reprend les grandes lignes d'un univers spatio-temporel désigné par un premier récit concernant la ville de Carthage et transporté une quinzaine de siècles plus tard avec les images qui symbolisent l'événement en lui-même : « le cri » de la destruction et de la souffrance, «le silence » du désespoir dû à la destruction de la ville. Ce même silence, on l'observe chez les esclaves du fait de l'épuisement et de l'angoisse face à l'avenir lors de leur arrivée dans le Nouveau monde :

Sous le silence écoute, ville, un flot un cri

À peine évanoui qui recommence, tu tressailles

La sève parmi toi ouvre une baie un vent un lys

Midi sculpte l'aride et l'éternel sur la hauteur.

Sel ô sel et blancheur, cœur de famine, impur labour 
Ombre nue que la femme a longtemps fuie ! Mot de la mer.

(Ibid. : 188) :

La reconquête de l'histoire se poursuit et Édouard Glissant fait du langage le théâtre de cette reconquête. Ainsi « le sel », tout en jouant sur la gamme de la «blancheur », apporte la mort. Répandu sur la terre à Carthage afin que plus rien ne (re)pousse et dans le but de susciter la famine au sein des populations, il est repris symboliquement dans le recueil Le Sel noir traduisant à juste titre l'image des esclaves morts pendant la traversée maritime et dont la trace et le souvenir réapparaissent sur les rives de l'archipel des Caraibes.

\section{L'emprise des mots et des images}

Selon Hölderlin, «c'est poétiquement que l'homme habite sur cette terre » (Hölderlin, 1967 : 939). Édouard Glissant et Derek Walcott ne dérogent pas à cette règle puisque leur réflexion ne se restreint pas aux seuls discours historique, géographique et biographique. Elle se rapporte également à l'écriture et c'est l'une de leurs priorités. Les poètes ont pour souci de rendre au mot sa puissance et de s'approprier l'imaginaire poétique. Quels sont les nouveaux procédés d'écriture dont ils font usage afin de réinvestir la mémoire?

L'un et l'autre expérimentent en effet la mémoire historique mais l'écriture se présente également comme le sujet de leur expérience. D'ailleurs, comment pourrait-il en être autrement, puisque l'écriture aux Antilles suppose cette contre-histoire révélatrice du système colonial et des autres systèmes de domination? Derek Walcott entretient avec sa langue un rapport décalé, révélateur de sa condition de poète antillais et de son identité créole. Il se réclame du calypso, forme de chanson populaire aux paroles satiriques, chanté dans une langue créole qui brasse des styles musicaux français, britanniques et espagnols et l'art africain. L'un des thèmes les plus importants de sa poétique est le sentiment de bipolarité faisant écho à son identité de métis.

Édouard Glissant ne déroge pas à cette règle mais, pour lui, il s'agit d'inventer une écriture qui puisse réconcilier l'intérieur et l'extérieur, la mémoire et le vécu quotidien. Si celle-ci requiert un important travail de la part de l'auteur, elle sollicite aussi un travail de la part du lecteur. Ce dernier est alors invité à percevoir les articulations, à reconnaître les tours et détours sémantiques de certains mots et à se familiariser avec tous les procédés issus de l'imagologie glissantienne. Édouard Glissant appelle donc à la réalisation d'un texte à partir duquel le lecteur découvre le discours caché ou les mystères qui circulent dans les mots et les nombreux jeux de relations qu'ils tissent, par exemple les jeux d'homophonie entre "la mer » et "la mère ». La formule qu'il utilise dans le vers en contre-rejet « La gonfle de cadavres, de prurits », extrait du Sel noir, attire l'attention, d'autant qu'elle renvoie à l'image d'une mer féconde, et on pourrait voir ici, dans un sens large, une représentation du renflement du ventre de la femme enceinte. Cependant, cette mer présage aussitôt la mort et une image maléfique, puisqu'elle porte en elle la trace des millions de «cadavres » qui ont été jetés par-dessus bord pendant la traversée :

La mer crie mais la mer bientôt s'éteint. Et le soldat

La gonfle de cadavres, de prurits. La mer accueille

Consentante le prix de son péage, elle est louée

Débiteuse de vie dont l'étal ébloui reflue...

(Glissant, 1994 : 186-187) 

matérielle d'une mémoire blessée et meurtrie, si bien qu'elle constitue un mythe dans la littérature caribéenne. On ne peut s'empêcher de penser au Chabin dans "The schooner flight » qui assimile la mer à une prison dont il tente inlassablement de s'échapper : « This time shabine like you really gone » (Walcott, 1992a : 8). L'écriture d'Édouard Glissant et de Derek Walcott n'est pas seulement expression, elle est aussi recherche et nécessité de s'informer de tout. La matérialisation de l'expérience mémorielle passe aussi par une pensée d'ouverture vers d'autres environnements culturels. La particularité de la réflexion glissantienne et walcottienne sur la question de la mémoire devient celle d'une globalité géographique. Ainsi l'histoire des petites Antilles ne se limite-t-elle plus à l'archipel des Caraïbes mais devient celle du «Tout-monde». On peut supposer que, compte tenu des caractères complexes de l'identité antillaise, marquée par diverses cultures du monde, il est difficile de penser toute entreprise mémorielle hors de l'histoire du monde. Édouard Glissant, par exemple, refuse toute notion d'abstraction et préfère parler de polysémie en rendant compte de multiples facettes de l'histoire antillaise, qui interfèrent avec d'autres histoires, voisines ou contraires. Il imagine de nouveaux enjeux concernant les histoires du monde dont l'histoire des Caraïbes n'est qu'un aspect. Il prône alors l'idée que toutes les histoires (tragiques ou non) doivent être reconnues et doivent faire sens pour tous. De même, la mémoire des peuples du monde englobe plusieurs faits et plusieurs personnalités qu'il relie à d'autres lieux dans une grande fresque animée par un jeu de parallélismes et de ressemblances.

Cette approche comparatiste montre en fin de compte que l'écriture de la mémoire n'est pas seulement ce qui est établi par des manuels historiques, par des chroniques ou autres registres d'état civil. Ce qui nous frappe dans l'écriture mémorielle de ces deux poètes, c'est qu'elle puise simultanément et paradoxalement dans l'oubli. N'est-ce pas parce qu'il y a oubli qu'on parle de reconstitution ? L'écriture de la mémoire chez Édouard Glissant et Derek Walcott n'est-elle pas en réalité l'écriture de l'oubli ? L'examen du fait mémoriel chez les poètes fait appel à une masse de matériaux qui prennent la forme tant de repères spatiaux que de données imaginaires et à partir desquels ils s'appliquent à oublier l'oubli. C'est en des termes neufs que les auteurs élaborent une grande mémoire, une mémoire en archipel à la fois plurielle et impersonnelle. Elle s'impose alors à tous, constituée de fragments culturels épars, d'effets de montages, d'éléments décontextualisés qui sont sans cesse réactualisés (parfois de manière obsessionnelle) dans l'unique but de sauver des cultures du risque de disparition ou d'amnésie.

Loin d'exprimer un repli communautaire sur ses propres valeurs, l'écriture de la mémoire antillaise est une pensée d'ouverture vers d'autres horizons culturels. Les auteurs s'adressent à toute l'humanité, d'où les diversités à l'intérieur de chacune des formes de pensée et d'écriture. Édouard Glissant et Derek Walcott s'approprient des langages poétiques occidentaux pour les associer à l'imaginaire caribéen et africain. En mettant en contact diverses cultures, ils affirment que chaque peuple peut combler ses propres lacunes mémorielles sans occulter l'histoire du monde. 


\section{BIBLIOGRAPHIE}

\section{Ouvrages}

Benjamin, Walter, 2000, « Sur le concept d'histoire » [1942], Euvres III, Paris, Gallimard, coll. « Folio essais », p. 427-443.

Chancé, Dominique, 2000, L'auteur en souffrance, Essai sur la position et la représentation de l'auteur dans le roman antillais contemporain (1981-1992), Paris, PUF.

Favre, Yves-Alain et Ferreira de Brito, Antonio (dir), 1992,Horizons d'Édouard Glissant, actes du colloque international organisé par le Centre de recherches sur la poésie contemporaine de l'université de Pau et le Département de français de l'université de Porto (octobre 1990), Pau, J \& D Éditions.

Genette, Gérard, 1982, Palimpsestes. La littérature au second degré, Paris, Seuil, coll. « Points ». Glissant, Édouard, 1981, Le Discours antillais, Paris, Seuil.

-, 1994, Poèmes complets, Paris, Gallimard.

-, 1997, La Case du commandeur, Paris, Seuil.

Hölderlin, Friedrich, 1967, CEuvres complètes, traductions Philippe Jaccottet, D. Naville, Gustave Roud, R. Rovini, François Fédier, Michel Deguy, André du Bouchet, Paris, Gallimard, coll. «Bibliothèque de La Pléiade ».

Lejeune, Philippe, 2001 [1975], Le Pacte autobiographique, Paris, Seuil, coll. « Points ».

Moulin, Joanny, 2005, The Collected Poems, Derek Walcott, Paris, Armand Colin-Cned.

Pageaux, Daniel-Henri, 2003, Trente essais de littérature générale et comparée ou la corne d'Amalthée, Paris, L'Harmattan.

Walcott, Derek, 1992a, The Star Apple-Kingdom, (Le Royaume du fruit-étoile), édition bilingue, traduction en français de Claire Malroux, Saulxures, Circé.

-, 1992b, Collected Poems 1948-1984, Londres, Faber and Faber.

-, 2004a, Café Martinique (What the twilight says), trad. Béatrice Dunner, Monaco, Éditions Du Rocher.

-, 2004b, Le Chien de Tiepolo : poème à Camille Pissarro (Tiepolo's Hound), trad. Marie-Claude Peugeot avec le concours du Centre national du livre, Monaco, Éditions Du Rocher.

\section{Articles}

Jacquart, Alain, 1992, « L'île dans l'œuvre d'Édouard Glissant », dans Yves-Alain Favre et Antonio Fereira de Brito (dir.), 1992 : 229-243.

Joubert, Jean-Louis, 2000, La littérature des Caraibes anglophones, Cd-rom Encyclopédie Universalis. 
Mathieu, François, « Poésie Derek Walcott, poète antillais », http://www.humanite.fr/ node/269793.

\section{NOTES}

1. «Jusqu'à ce qu'une femme noire, au capuchon de busard, gravisse l'escalier et vienne frapper à la porte de son rêve, chuchotant à l'oreille de la serrure: "Ouvre-moi, fini les prières, je suis la Révolution. Je suis l'Amérique la plus sombre, la plus ancienne”. Elle était aussi belle qu'un roc à l'aurore, sa voix crépitait des gutturales de mitraillettes » (Ibid:95).

2. «Elle lui caressa les cheveux jusqu'à les faire blanchir, mais elle refusait de comprendre qu'il ne voulait pas d'autre pouvoir que la paix, qu'il voulait une révolution sans effusion de sang; il voulait une histoire sans mémoire, des rues sans statues, une géographie sans mythe. Il ne voulait pas d'armées, mais des régimes de bananes, les lances épaisses des cannes, et il pleura, disant: "Je n'ai pas de pouvoir, sauf l'amour" (Ibid. : 97).

3. «Je vois de grands amiraux, / Rodney, Nelson, de Grasse, j'entends les ordres rauques / qu'ils lancent aux Chabins, et cette forêt de mats / navigue en plein à travers le Flight, / on n'entend rien que la rumeur fantomatique / des vagues bruissant comme l'herbe dans la brise / et le crissement des algues traînées à la poupe, / lentement ils virent d'est en ouest » (Ibid. : 23).

\section{RÉSUMÉS}

"C'est toujours la même histoire ». Cette expression pourrait être significative pour celui qui étudie les écrits de Glissant et de Walcott. Pourquoi ? Parce que même aujourd'hui, l'histoire suscite encore de nombreuses questions dans la région caribéenne. Pour de nombreux autochtones des Antilles, par exemple, le problème historique provoque des souffrances psychologiques. C'est la raison pour laquelle de nombreux écrivains autochtones ont abordé le problème de l'identité. Ils doivent affronter des stéréotypes et d'autres expressions traditionnelles, comme « nos ancêtres les Gaulois » que l'on a utilisées quand on parlait des Noirs pendant l'Ère de l'esclavage. C'est pour cette raison que l'histoire reste problématique de nos jours. Dans leurs œuvres, ces auteurs se réfèrent à, ou rendent compte de beaucoup d'événements dramatiques. Chaque poème transmet les sentiments du poète: un intérêt passionné dans certains cas mais aussi de la défiance dans d'autres. La poésie de Glissant et la poésie de Walcott révèlent toujours une attention particulière portée au processus historique. Comme leur poésie, surtout celle de Glissant, se base sur de nombreuses réflexions théoriques, leurs écrits affrontent le problème de savoir si l'écriture peut traduire la réalité de l'histoire. L'imagination et le paysage se mêlent pour recréer l'histoire. Qui plus est, en accordant une attention particulière à la dimension subjective des souvenirs historiques, les écrivains remettent en question la représentation du passé, un passé qui ressemble beaucoup à un palimpseste puisque chaque fragment de souvenir fait partie de la texture de l'histoire.

"It's the same old story". This phrase could be significant when studying Glissant's and Walcott's works. Why? Because even now history still raises many questions in the Caribbean area. For many native people from the West Indies, for instance, the historical problem induces psychological suffering. That is why many native writers have addressed the problem of identity. 
They have to deal with stereotypes and other traditional expressions such as "vos ancêtres les Gaulois", that were used when referring to Black people during the Age of slavery. That is why history nowadays remains problematic. In their works, these authors refer to, or give an account of, many dramatic events. Each poem conveys the feelings of the poet: a passionate interest in some cases, but also mistrust in others. Glissant's and Walcott's poetry always reveals a particular attention to the historical process. As their poetry, especially Glissant's, is based on many theoretical refiections, their works tackle the problem of knowing whether writing is able to translate the reality of history. Imagination and landscape mingle to recreate history. What is more, as they pay particular attention to the subjective dimension of historical memories, the writers question the representation of the past: a past that looks much like a palimpsest, because each fragment of memory is part of the texture of history.

INDEX

Mots-clés : mémoire, oubli, identité, Antilles, langage

Keywords : memory, oblivion, identity, Caribbean area, language

\section{AUTEUR}

AMINATA KEITA

Université Paris 3 